\title{
New Even Levels and Classified Lines in the First Spectrum of Tungsten ( $W_{1}$ )
}

\author{
C. H. Corliss \\ Institute for Basic Standards, National Bureau of Standards, Washington, D.C. 20234
}

(January 24, 1969)

\begin{abstract}
Ten new even levels, which classify 161 previously unclassified lines, have been found in the first spectrum of tungsten. Nine of these fit levels in the $(5 d+6 s)^{6}$ mixture of configurations calculated theoretically by Shadmi and Caspi.

Key words: Atomic energy levels in W I; classified lines of W I; spectrum, W I; tungsten, first spectrum; W I.
\end{abstract}

Recently Laun and Corliss [1968] published an extensive list of levels and classified lines for the first spectrum of tungsten (W I). Prompted by these experimental data, Shadmi and Caspi [1968] published a theoretical interpretation of the low even levels arising from the $(5 d+6 s)^{6}$ mixture of configurations. Their work provided an interpretation for every level found experimentally below $40000 \mathrm{~cm}^{-1}$ and predicted values for numerous levels not yet found. It seemed worthwhile to search for some of these levels.

The search has been made with the Combo program for finding energy levels, which was developed by J. L. Tech in the National Bureau of Standards Spectroscopy Section. With this program, the wavenumbers of 1391 unclassified $W_{\text {I }}$ lines were used to search for new even levels in the energy region where levels were predicted to lie. Ten new levels with numbers of combinations significantly above the noise were found. (The noise level in this case was about 6 to 8 combinations for fortuitous levels.) The new levels, which have $J$-values $2,3,4$, and 5 are marked with asterisks $(*)$ in table 1 .

The previously known levels with $J$-values from 1 through 5 are also listed in order to show the remarkable regularity in the relationship between the number of combinations $(N)$ made by each level and the level value. According to Shadmi and Caspi, the three levels marked in parentheses seem of doubtful reality, in view of the relatively large differences between the observed and calculated values for these levels. However, the tabulations in table 1 show that the number of combinations made by these three levels in question is about the number to be expected. Furthermore, since that number is about five times above the noise level number, it is likely that the levels are indeed real.
TABLE 1. Even levels in W I.

\begin{tabular}{|c|c|c|c|c|}
\hline $\begin{array}{l}\text { Number of } \\
\text { combinations }\end{array}$ & $J$ & $\begin{array}{c}\text { Observed level } \\
\left(\mathrm{cm}^{-1}\right)\end{array}$ & $\begin{array}{l}\text { Calculated } \\
\text { level }\left(\mathrm{cm}^{-1}\right)\end{array}$ & $\begin{array}{l}\text { Difference } \\
\text { O-C }\left(\mathrm{cm}^{-1}\right)\end{array}$ \\
\hline 87 & 1 & 1670.29 & 1697 & -27 \\
\hline 103 & 1 & 13307.10 & 13405 & -98 \\
\hline 84 & 1 & 18082.83 & 18095 & -12 \\
\hline 78 & 1 & 20427.84 & 20407 & 21 \\
\hline 58 & 1 & 23455.02 & 23541 & -86 \\
\hline 34 & 1 & $(27670.48)$ & 28123 & $(-453)$ \\
\hline 24 & 1 & 28720.88 & 28877 & -156 \\
\hline 15 & 1 & 32378.40 & 32443 & -65 \\
\hline 128 & 2 & 3325.53 & 3382 & -57 \\
\hline 144 & 2 & 13777.71 & 13708 & 70 \\
\hline 149 & 2 & 14976.18 & 15058 & -82 \\
\hline 131 & 2 & 18116.84 & 18240 & -123 \\
\hline 132 & 2 & 18280.48 & 18385 & -105 \\
\hline 130 & 2 & 19253.56 & 19283 & -30 \\
\hline 110 & 2 & 20983.02 & 21031 & -48 \\
\hline 77 & 2 & 23982.80 & 24028 & -45 \\
\hline 57 & 2 & 24789.66 & 24799 & -9 \\
\hline 61 & 2 & 26861.64 & 26844 & 18 \\
\hline 44 & 2 & (28204.20) & 28576 & $(-372)$ \\
\hline 34 & 2 & 28898.96 & 28790 & 109 \\
\hline 30 & 2 & $(30374.20)$ & 29840 & $(534)$ \\
\hline 18 & 2 & *31077.79 & 31037 & 41 \\
\hline 107 & 3 & 2951.29 & 2833 & 118 \\
\hline 145 & 3 & 4830.00 & 4836 & -6 \\
\hline 173 & 3 & 13348.56 & 13424 & -76 \\
\hline 173 & 3 & 15460.01 & 15405 & 55 \\
\hline 154 & 3 & 17701.18 & 17736 & -35 \\
\hline 152 & 3 & 18974.51 & 18994 & -20 \\
\hline 154 & 3 & 19827.68 & 19759 & 69 \\
\hline 114 & 3 & 23930.08 & 23870 & 60 \\
\hline 85 & 3 & 24610.88 & 24741 & -130 \\
\hline 62 & 3 & 28291.88 & 28196 & 96 \\
\hline 54 & 3 & 28347.60 & 28454 & -106 \\
\hline 26 & 3 & $*_{2} 29430.50$ & 29409 & 21 \\
\hline 17 & 3 & *32217.95 & 31996 & 222 \\
\hline
\end{tabular}


TABLE 1. Even levels in W I. - Continued

\begin{tabular}{|c|c|c|c|c|c|c|c|c|c|}
\hline $\begin{array}{c}\text { Number of } \\
\text { combinations }\end{array}$ & $J$ & $\begin{array}{c}\text { Observed level } \\
\left(\mathrm{cm}^{-1}\right)\end{array}$ & $\begin{array}{l}\text { Calculated } \\
\text { level }\left(\mathrm{cm}^{-1}\right)\end{array}$ & $\begin{array}{l}\text { Difference } \\
\mathrm{O}-\mathrm{C}\left(\mathrm{cm}^{-1}\right)\end{array}$ & $\begin{array}{l}\text { Number of } \\
\text { combinations }\end{array}$ & $J$ & $\begin{array}{c}\text { Observed level } \\
\left(\mathrm{cm}^{-1}\right)\end{array}$ & $\begin{array}{l}\text { Calculated } \\
\text { level }\left(\mathrm{cm}^{-1}\right)\end{array}$ & $\begin{array}{l}\text { Difference } \\
\mathrm{O}-\mathrm{C}\left(\mathrm{cm}^{-1}\right)\end{array}$ \\
\hline 13 & 3 & *32826.63 & 32821 & 6 & 50 & 4 & 29853.66 & 29813 & 41 \\
\hline 11 & 3 & *33952.85 & 33675 & 278 & 31 & 4 & 32135.94 & 31902 & 234 \\
\hline 10 & 3 & ${ }^{*} 34465.83$ & 34566 & -100 & 17 & 4 & *33569.53 & 33421 & 149 \\
\hline 10 & 3 & *37414.11 & 37143 & 271 & 26 & 4 & $34302 \div 04$ & 34159 & 143 \\
\hline & & & & & 14 & 4 & *35299.82 & 35219 & 81 \\
\hline 133 & 4 & 6219.33 & 6105 & 114 & & & & & \\
\hline 159 & 4 & 12161.96 & 12162 & 0 & 123 & 5 & 15069.93 & 15124 & -54 \\
\hline 144 & 4 & 16431.31 & 16567 & -136 & 115 & 5 & 19535.01 & 19391 & $144^{\circ}$ \\
\hline 153 & 4 & 17107.0 & 1704 & 64 & 115 & 5 & 19826.04 & 20035 & -209 \\
\hline 138 & 4 & 19256.24 & 19275 & -19 & 68 & 5 & 27849.80 & 27755 & 95 \\
\hline 117 & 4 & 22476.68 & 22442 & 35 & 67 & 5 & 28233.44 & 28273 & -40 \\
\hline 99 & 4 & 22852.80 & 22826 & 27 & 49 & 5 & 31389.08 & 31256 & 133 \\
\hline 81 & 4 & 27213.82 & 27114 & 100 & 20 & 5 & *33201.61 & & \\
\hline 48 & 4 & 29479.32 & 29453 & 26 & 28 & 5 & 33291.80 & 33352 & -60 \\
\hline
\end{tabular}

TABLE 2.-Newly Classified Lines of $\mathrm{W}_{\mathrm{I}}$

\begin{tabular}{|c|c|c|c|c|c|c|c|c|c|}
\hline \multirow{2}{*}{$\begin{array}{l}\text { Wave- } \\
\text { length } \\
\AA\end{array}$} & \multirow{2}{*}{$\begin{array}{l}\text { Inten- } \\
\text { sity }\end{array}$} & \multicolumn{2}{|c|}{ Wavenumber $\left(\mathrm{cm}^{-1}\right)$} & \multirow{2}{*}{$\begin{array}{c}\text { Classification } \\
\text { (levels in } \mathrm{cm}^{-1} \text { ) }\end{array}$} & \multirow{2}{*}{$\begin{array}{c}\text { Wave- } \\
\text { length } \\
\text { A }\end{array}$} & \multirow{2}{*}{$\begin{array}{l}\text { Inten- } \\
\text { sity }\end{array}$} & \multicolumn{2}{|c|}{ Wavenumber $\left(\mathrm{cm}^{-1}\right)$} & \multirow{2}{*}{$\begin{array}{l}\text { Classification } \\
\text { (levels in } \mathrm{cm}^{-1} \text { ) }\end{array}$} \\
\hline & & Observed & $\mathrm{O}-\mathrm{C}$ & & & & Observed & $\mathrm{O}-\mathrm{C}$ & \\
\hline 3113.876 & 8 & 32105.01 & 0.01 & $29430_{3}-61535_{4}^{\circ}$ & 4736.036 & 3 & 21108.80 & 0.11 & $332015-54310_{5}^{\circ}$ \\
\hline 3523.446 & 3 & 28373.19 & 0.03 & $29430_{3}-57803_{4}^{\circ}$ & 4809.642 & 3 & 20785.76 & -0.05 & $32217_{3}-53003_{2}^{\circ}$ \\
\hline 3578.011 & 2 & 27940.51 & 0.10 & $32217_{3}-60158^{\circ}$ & 4865.33 & 3 & 20547.85 & 0.02 & $33569_{4}-54117^{\circ}$ \\
\hline 3580.40 & 2 & 27921.87 & 0.05 & $29430_{3}-57352_{3}^{\circ}$ & 5019.52 & 3 & 19916.67 & -0.01 & $33201-53118^{\circ}$ \\
\hline 3608.904 & 4 & 27701.34 & 0.06 & $29430_{3}-57131_{3}^{\circ}$ & 5023.423 & $2 b$ & 19901.19 & 0.05 & $37414_{3}-57315^{\circ}$ \\
\hline 3625.059 & 2 & 27577.90 & -0.04 & $31077_{2}-58655_{2}^{\circ}$ & 5044.908 & 4 & 19816.44 & 0.11 & $31077_{2}-50894_{3}^{\circ}$ \\
\hline 3627.611 & 2 & 27558.50 & 0.04 & $32826_{3}-60385_{4}^{\circ}$ & 5094.173 & 6 & 19624.80 & 0.08 & $33569_{4}-53194^{\circ}$ \\
\hline 3726.776 & 2 & 26825.22 & 0.07 & $29430_{3}-56255^{\circ}$ & 5107.78 & 5 & 19572.52 & 0.03 & $33201-52774^{\circ}$ \\
\hline 3749.935 & 2 & 26659.55 & -0.10 & $33569_{4}-60229_{5}^{\circ}$ & 5133.11 & 20 & 19475.94 & 0.00 & $32217_{3}-51693_{2}^{\circ}$ \\
\hline 3918.195 & 2 & 25514.73 & 0.18 & $32217_{3}-57732_{3}^{\circ}$ & 5145.453 & 8 & 19429.22 & 0.05 & $32826_{3}-52255_{3}^{\circ}$ \\
\hline 3931.44 & 2 & 25428.77 & 0.12 & $29430_{3}-54859^{\circ}$ & 5282.646 & $4 b$ & 18924.64 & 0.05 & $34465_{3}-53390_{3}^{\circ}$ \\
\hline 3978.822 & 2 & 25125.96 & 0.03 & $29430_{3}-54556_{3}^{\circ}$ & 5295.252 & 6 & 18879.59 & 0.11 & $33201 ;-52081^{\circ}$ \\
\hline 4005.893 & 8 & 24956.17 & 0.00 & $34465_{3}-59422_{2}^{\circ}$ & 5295.252 & 6 & 18879.59 & -0.10 & $34465_{3}-53345_{3}^{\circ}$ \\
\hline 4012.704 & 1 & 24913.81 & -0.06 & $32217_{3}-57131_{3}^{\circ}$ & 5310.37 & 4 & 18825.84 & -0.13 & $33569_{4}-52395^{\circ}$ \\
\hline 4048.854 & 5 & 24691.37 & 0.12 & $33952_{3}-58644_{3}^{\circ}$ & 5312.282 & 5 & 18819.07 & 0.11 & $35299_{4}-54118^{\circ}$ \\
\hline 4062.26 & 1 & 24609.89 & 0.04 & $33569_{4}-58179_{5}^{\circ}$ & 5312.282 & 5 & 18819.07 & -0.02 & $34465_{3}-53284_{2}^{\circ}$ \\
\hline 4101.648 & 3 & 24373.57 & 0.07 & $35299_{4}-59673^{\circ}$ & 5345.887 & $3 b$ & 18700.77 & 0.04 & $33952_{3}-52653_{2}^{\circ}$ \\
\hline 4116.718 & 3 & 24284.34 & 0.15 & $32217_{3}-56502^{\circ}$ & 5389.661 & 3 & 18548.88 & 0.08 & $35299_{4}-53848^{\circ}{ }_{4}$ \\
\hline 4158.954 & 2 & 24037.73 & -0.01 & $32217_{3}-56255^{\circ}$ & 5392.78 & 4 & 18538.16 & 0.04 & $29430_{3}-47968_{4}^{\circ}$ \\
\hline 4167.002 & $2 b$ & 23991.30 & 0.01 & $33569_{4}-57560^{\circ}$ & 5443.35 & 12 & 18365.94 & 0.02 & $31077_{2}-49443^{\circ}$ \\
\hline 4240.913 & 3 & 23573.19 & -0.03 & $29430_{3}-53003_{2}^{\circ}$ & 5469.932 & 2 & 18276.68 & 0.05 & $32217_{3}-50494_{2}^{\circ}$ \\
\hline 4257.978 & 3 & 23478.72 & 0.08 & $31077_{2}-54556^{\circ}$ & 5471.50 & 3 & 18271.45 & 0.02 & $33201 ;-51473^{\circ}$ \\
\hline 4262.267 & 4 & 23455.09 & 0.09 & $29430_{3}-52885_{2}^{\circ}$ & 5479.229 & 10 & 18245.67 & 0.17 & $32826_{3}-51072_{3}^{\circ}$ \\
\hline 4282.922 & 1 & 23341.98 & 0.10 & $31077_{2}-54419^{\circ}$ & 5495.256 & 3 & 18192.46 & 0.09 & $31077_{2}-49270_{2}^{\circ}$ \\
\hline 4290.53 & 2 & 23300.59 & 0.10 & $33201 ;-56502^{\circ}$ & 5526.644 & $2 b$ & 18089.14 & 0.02 & $332015-51290_{5}^{\circ}$ \\
\hline 4314.424 & 2 & 23171.55 & 0.16 & $32217_{3}-55389_{3}^{\circ}$ & 5539.91 & 2 & 18045.82 & 0.12 & $35299_{4}-53345_{3}^{\circ}$ \\
\hline 4352.11 & $2 b$ & 22970.90 & -0.08 & $37414_{3}-60385_{4}^{\circ}$ & 5550.319 & 4 & 18011.98 & -0.05 & $29430_{3}-47442_{2}^{\circ}$ \\
\hline 4359.37 & 2 & 22932.65 & 0.08 & $33569_{+}-56502_{+}^{\circ}$ & 5563.97 & $4 b$ & 17967.79 & 0.00 & $32217_{3}-50185_{3}^{\circ}$ \\
\hline 4364.40 & 3 & 22906.22 & 0.04 & $35299_{4}-58206_{3}^{\circ}$ & 5641.39 & 5 & 17721.21 & 0.01 & $33569_{4}-51290^{\circ}$ \\
\hline 4381.862 & 5 & 22814.94 & 0.18 & $32217_{3}-55032_{2}^{\circ}$ & 5721.10 & 2 & 17474.31 & 0.03 & $35299_{4}-52774_{5}^{\circ}$ \\
\hline 4509.30 & $4 b$ & 22170.17 & 0.15 & $29430_{3}-51600_{3}^{\circ}$ & 5726.43 & 2 & 17458.04 & 0.03 & $32826_{3}-50284_{4}^{\circ}$ \\
\hline 4535.389 & 2 & 22042.64 & 0.10 & $29430:-51473_{4}^{\circ}$ & 5803.04 & 3 & 17227.57 & 0.00 & $33201 ;-50429^{\circ}$ \\
\hline 4584.244 & 3 & 21807.74 & 0.03 & $31077_{2}-52885_{2}^{\circ}$ & 5848.81 & 2 & 17092.76 & 0.00 & $31077_{2}-48170_{3}^{\circ}$ \\
\hline 4731.027 & 2 & 21131.15 & -0.02 & $33952_{3}-55084_{2}^{\circ}$ & 5905.05 & 4 & 16929.97 & -0.07 & $32217_{3}-49147^{\circ}$ \\
\hline 4731.027 & 2 & 21131.15 & 0.08 & $34465_{3}-55596_{4}^{\circ}$ & 5931.13 & 2 & 16855.52 & -0.14 & $37414_{3}-54269^{\circ}$ \\
\hline
\end{tabular}


TABLE 2.-Newly Classified Lines of $\mathrm{W}_{\mathrm{I}}$ - Continued

\begin{tabular}{|c|c|c|c|c|c|c|c|c|c|}
\hline \multirow{2}{*}{$\begin{array}{l}\text { Wave- } \\
\text { length } \\
\AA\end{array}$} & \multirow{2}{*}{$\begin{array}{l}\text { Inten- } \\
\text { sity }\end{array}$} & \multicolumn{2}{|c|}{ Wavenumber $\left(\mathrm{cm}^{-1}\right)$} & \multirow{2}{*}{$\begin{array}{l}\text { Classification } \\
\text { (levels in } \mathrm{cm}^{-1} \text { ) }\end{array}$} & \multirow{2}{*}{$\begin{array}{l}\text { Wave- } \\
\text { length } \\
\text { A }\end{array}$} & \multirow{2}{*}{$\begin{array}{l}\text { Inten- } \\
\text { sity }\end{array}$} & \multicolumn{2}{|c|}{ Wavenumber $\left(\mathrm{cm}^{-1}\right)$} & \multirow{2}{*}{$\begin{array}{c}\text { Classification } \\
\text { (levels in } \mathrm{cm}^{-1} \text { ) }\end{array}$} \\
\hline & & Observed & $\mathrm{O}-\mathrm{C}$ & & & & Observed & $\mathrm{O}-\mathrm{C}$ & \\
\hline $\begin{array}{l}5980.95 \\
6053.17 \\
6082.89 \\
6179.66 \\
6181.39\end{array}$ & $\begin{array}{l}2 \\
2 b \\
3 \\
3 \\
2\end{array}$ & $\begin{array}{l}16715.12 \\
16515.70 \\
16435.00 \\
16177.64 \\
16173.12\end{array}$ & $\begin{array}{r}0.01 \\
0.05 \\
0.09 \\
-0.12 \\
-0.10\end{array}$ & $\begin{array}{l}33569_{4}-50284_{4}^{\circ} \\
31077_{2}-47593_{3}^{\circ} \\
33201_{5}-49636_{4}^{\circ} \\
31077_{2}-47255^{\circ} \\
35299_{4}-51473_{4}^{\circ}\end{array}$ & $\begin{array}{l}7245.88 \\
7318.95 \\
7416.29 \\
7416.29 \\
7473.90\end{array}$ & $\begin{array}{l}1 \\
1 \\
1 \\
1 \\
1\end{array}$ & $\begin{array}{l}13797.14 \\
13659.40 \\
13480.12 \\
13480.12 \\
13376.21\end{array}$ & $\begin{array}{r}-0.02 \\
-0.02 \\
-0.12 \\
0.11 \\
-0.05\end{array}$ & $\begin{array}{l}29430_{3}-43227^{\circ}{ }_{2} \\
31077_{2}-44737^{\circ}{ }_{1} \\
29430_{3}-42910^{\circ}{ }_{4} \\
37414_{3}-50894^{\circ}{ }_{3} \\
35299_{4}-48676^{\circ}\end{array}$ \\
\hline $\begin{array}{l}6243.10 \\
6251.48 \\
6253.63 \\
6269.28 \\
6298.57\end{array}$ & $\begin{array}{l}2 \\
5 \\
6 \\
4 \\
6\end{array}$ & $\begin{array}{l}16013.25 \\
15991.79 \\
15986.29 \\
15946.38 \\
15872.23\end{array}$ & $\begin{array}{r}0.07 \\
0.03 \\
-0.02 \\
0.04 \\
-0.04\end{array}$ & $\begin{array}{l}33952_{3}-49966^{\circ} \\
294300_{3}-45422^{\circ}{ }_{2} \\
33201_{5}-49187^{\circ} \\
33201_{5}-49147^{\circ} \\
33201_{5}-49073_{5}^{\circ}\end{array}$ & $\begin{array}{l}7590.55 \\
7641.04 \\
7678.57 \\
7680.12 \\
7702.63\end{array}$ & $\begin{array}{l}1 \\
3 \\
6 \\
1 \\
2 b\end{array}$ & $\begin{array}{l}13170.65 \\
13083.62 \\
13019.68 \\
13017.05 \\
12979.01\end{array}$ & $\begin{array}{r}-0.04 \\
-0.02 \\
-0.06 \\
-0.02 \\
0.02\end{array}$ & $\begin{array}{l}29430_{3}-42601_{3}^{\circ} \\
29430_{3}-42514^{\circ}{ }_{3} \\
29430_{3}-42450^{\circ}{ }_{4} \\
21448_{2}^{\circ}-34465_{3} \\
33952_{3}-46931_{4}^{\circ}\end{array}$ \\
\hline $\begin{array}{l}6415.03 \\
6417.36 \\
6447.25 \\
6448.76 \\
6449.93\end{array}$ & $\begin{array}{l}1 \\
4 \\
1 \\
1 \\
3\end{array}$ & $\begin{array}{l}15584.08 \\
15578.43 \\
15506.20 \\
15502.57 \\
15499.76\end{array}$ & $\begin{array}{r}0.04 \\
0.01 \\
-0.05 \\
-0.04 \\
-0.02\end{array}$ & $\begin{array}{l}29430_{3}-45014_{3}^{\circ} \\
33569_{4}-49147^{\circ}{ }_{4} \\
35299_{4}-50806_{5}^{\circ} \\
33569_{4}-49072_{3}^{\circ} \\
32826_{3}-48326_{3}^{\circ}\end{array}$ & $\begin{array}{l}7704.00 \\
7747.07 \\
7797.57 \\
7812.44 \\
7826.86\end{array}$ & $\begin{array}{l}1 b \\
3 \\
5 \\
7 \\
3\end{array}$ & $\begin{array}{l}12976.70 \\
12904.56 \\
12820.98 \\
12796.58 \\
12773.00\end{array}$ & $\begin{array}{r}0.00 \\
-0.04 \\
-0.03 \\
-0.05 \\
-0.05\end{array}$ & $\begin{array}{l}34465_{3}-47442^{\circ}{ }_{2}^{\circ} \\
33201_{5}-46106_{6}^{\circ} \\
29430_{3}-42251^{\circ}{ }_{3} \\
32217_{3}-45014_{3}^{\circ}{ }_{3} \\
31077_{2}-43850_{3}^{\circ}\end{array}$ \\
\hline $\begin{array}{l}6461.78 \\
6461.78 \\
6464.43 \\
6515.44 \\
6602.37\end{array}$ & $\begin{array}{l}5 \\
5 \\
4 \\
3 \\
1\end{array}$ & $\begin{array}{l}15471.34 \\
15471.34 \\
15464.99 \\
15343.92 \\
15141.89\end{array}$ & $\begin{array}{l}-0.08 \\
-0.05 \\
-0.06 \\
-0.01 \\
-0.10\end{array}$ & $\begin{array}{l}32217_{3}-47689^{\circ}{ }_{4} \\
37414_{3}-52885^{\circ} \\
33952_{3}-49417^{\circ}{ }_{3} \\
32826_{3}-48170^{\circ} \\
32826_{3}-47968^{\circ}\end{array}$ & $\begin{array}{l}7967.14 \\
7998.74 \\
8039.04 \\
8061.78 \\
8076.41\end{array}$ & $\begin{array}{r}20 \\
2 \\
1 \\
2 b \\
2\end{array}$ & $\begin{array}{l}12548.10 \\
12498.53 \\
12435.88 \\
12400.80 \\
12378.34\end{array}$ & $\begin{array}{r}-0.02 \\
0.04 \\
-0.04 \\
0.01 \\
-0.03\end{array}$ & $\begin{array}{l}29430_{3}-41978^{\circ} \\
33569_{4}-46068^{\circ} \\
32826_{3}-45262^{\circ} \\
31077_{2}-43478^{\circ} \\
32217_{3}-44596^{\circ}\end{array}$ \\
\hline $\begin{array}{l}6612.24 \\
6652.98 \\
6669.22 \\
6671.59 \\
6726.42\end{array}$ & $\begin{array}{l}2 \\
1 \\
2 \\
1 \\
4\end{array}$ & $\begin{array}{l}15119.29 \\
15026.71 \\
14990.12 \\
14984.79 \\
14862.65\end{array}$ & $\begin{array}{r}0.00 \\
-0.12 \\
-0.11 \\
-0.03 \\
-0.06\end{array}$ & $\begin{array}{l}33952_{3}-49072_{3}^{\circ} \\
31077_{2}-46104^{\circ}{ }_{1} \\
31077_{2}-46068^{\circ}{ }_{3} \\
35299_{4}-50284_{4}^{\circ} \\
32826_{3}-47689^{\circ}\end{array}$ & $\begin{array}{l}8101.05 \\
8125.47 \\
8137.38 \\
8151.80 \\
8161.04\end{array}$ & $\begin{array}{r}1 \\
10 \\
8 \\
1 \\
1\end{array}$ & $\begin{array}{l}12340.68 \\
12303.60 \\
12285.59 \\
12263.86 \\
12249.97\end{array}$ & $\begin{array}{r}0.06 \\
-0.03 \\
0.14 \\
0.02 \\
-0.07\end{array}$ & $\begin{array}{l}34465_{3}-46806^{\circ}{ }_{2}^{\circ} \\
29430_{3}-41734_{2}^{\circ} \\
37414_{3}-49699^{\circ}{ }_{2} \\
29430_{3}-41694_{3}^{\circ} \\
33201_{5}-45451_{5}^{\circ}\end{array}$ \\
\hline $\begin{array}{l}6770.04 \\
6846.90 \\
6846.90 \\
6852.84 \\
6862.07\end{array}$ & $\begin{array}{l}8 \\
1 \\
1 \\
2 \\
5\end{array}$ & $\begin{array}{l}14766.89 \\
14601.12 \\
14601.12 \\
14588.46 \\
14568.84\end{array}$ & $\begin{array}{r}0.08 \\
0.10 \\
-0.05 \\
-0.08 \\
-0.01\end{array}$ & $\begin{array}{l}32826_{3}-47593_{3}^{\circ} \\
33569_{4}-48170_{3}^{\circ} \\
37414_{3}-52015^{\circ}{ }_{3} \\
32217_{3}-46806_{2}^{\circ} \\
33569_{4}-48138_{5}^{\circ}\end{array}$ & $\begin{array}{l}8175.02 \\
8181.34 \\
8251.89 \\
8283.50 \\
8289.01\end{array}$ & $\begin{array}{l}1 \\
1 \\
1 \\
2 \\
1\end{array}$ & $\begin{array}{l}12229.02 \\
12219.58 \\
12115.11 \\
12068.87 \\
12060.85\end{array}$ & $\begin{array}{l}-0.09 \\
-0.03 \\
-0.06 \\
-0.06 \\
-0.09\end{array}$ & $\begin{array}{l}32217_{3}-44447^{\circ}{ }_{3} \\
33569_{4}-45789^{\circ}{ }_{5} \\
33952_{3}-46068^{\circ}{ }_{3} \\
294300_{3}-41499^{\circ}{ }_{3} \\
33201_{5}-45262^{\circ}{ }_{4}\end{array}$ \\
\hline $\begin{array}{l}6900.54 \\
6971.64 \\
6992.96 \\
7031.56 \\
7033.14\end{array}$ & $\begin{array}{r}12 \\
15 \\
10 \\
2 \\
2\end{array}$ & $\begin{array}{l}14487.62 \\
14339.87 \\
14296.15 \\
14217.67 \\
14214.48\end{array}$ & $\begin{array}{l}-0.10 \\
-0.08 \\
-0.06 \\
-0.03 \\
-0.04\end{array}$ & $\begin{array}{l}33201_{5}-47689^{\circ}{ }_{4} \\
33201_{5}-47541_{6}^{\circ} \\
31077_{2}-45374^{\circ}{ }_{1} \\
33952_{3}-48170_{3} \\
35299_{4}-49514_{3}^{\circ}\end{array}$ & $\begin{array}{l}8390.39 \\
8428.10 \\
8470.08 \\
8495.70 \\
8619.49\end{array}$ & $\begin{array}{r}10 \\
1 \\
4 \\
8 \\
2\end{array}$ & $\begin{array}{l}11915.12 \\
11861.81 \\
11803.02 \\
11767.43 \\
11598.43\end{array}$ & $\begin{array}{l}-0.04 \\
-0.11 \\
-0.07 \\
-0.07 \\
-0.04\end{array}$ & $\begin{array}{l}33201_{5}-45116^{\circ}{ }_{4} \\
34465_{3}-46327^{\circ}{ }_{2} \\
32217_{3}-44021^{\circ}{ }_{3} \\
32217_{3}-43985^{\circ}{ }_{4} \\
33952_{3}-45551^{\circ}{ }_{3}\end{array}$ \\
\hline $\begin{array}{l}7043.94 \\
7060.86 \\
7081.21 \\
7098.22 \\
7128.71\end{array}$ & $\begin{array}{l}4 b \\
5 \\
1 \\
15 \\
2\end{array}$ & $\begin{array}{l}14192.69 \\
14158.68 \\
14117.99 \\
14084.15 \\
14023.92\end{array}$ & $\begin{array}{r}0.02 \\
0.10 \\
-0.09 \\
-0.03 \\
0.01\end{array}$ & $\begin{array}{l}37414_{3}-51606_{2}^{\circ} \\
33201_{5}-47360^{\circ}{ }_{4} \\
35299_{4}-49417_{3}^{\circ} \\
29430_{3}-43514^{\circ}{ }_{2} \\
33569_{4}-47593^{\circ}\end{array}$ & $\begin{array}{l}8651.92 \\
8662.53 \\
8691.03 \\
8698.35 \\
8791.89\end{array}$ & $\begin{array}{l}1 \\
1 \\
1 b \\
8 \\
1\end{array}$ & $\begin{array}{l}11554.95 \\
11540.80 \\
11502.96 \\
11493.28 \\
11371.00\end{array}$ & $\begin{array}{r}-0.03 \\
-0.07 \\
0.00 \\
-0.05 \\
-0.04\end{array}$ & $\begin{array}{l}35299_{4}-46854^{\circ} \\
32826_{3}-44367^{\circ} \\
32217_{3}-43720^{\circ} \\
29430_{3}-40923^{\circ} \\
33569_{4}-44940^{\circ}\end{array}$ \\
\hline $\begin{array}{l}7132.89 \\
7151.20 \\
7173.38 \\
7218.22 \\
7219.18\end{array}$ & $\begin{array}{l}1 \\
1 \\
5 b \\
6 b \\
2\end{array}$ & $\begin{array}{l}14015.70 \\
13979.81 \\
13936.59 \\
13850.01 \\
13848.17\end{array}$ & $\begin{array}{l}-0.07 \\
-0.01 \\
-0.17 \\
-0.10 \\
0.04\end{array}$ & $\begin{array}{l}33952_{3}-47968^{\circ}{ }_{4} \\
32826_{3}-46806^{\circ}{ }_{2} \\
31077_{2}-45014^{\circ}{ }_{3} \\
32217_{3}-46068^{\circ}{ }_{3} \\
35299_{4}-49147_{4}^{\circ}\end{array}$ & $\begin{array}{l}8947.10 \\
9018.33 \\
9270.69 \\
9353.72 \\
9928.10 \\
9968.85\end{array}$ & $\begin{array}{l}1 \\
1 \\
1 \\
2 \\
5\end{array}$ & $\begin{array}{l}11173.74 \\
11085.48 \\
10783.72 \\
10688.00 \\
10069.66 \\
10028.50\end{array}$ & $\begin{array}{r}0.02 \\
-0.01 \\
-0.08 \\
-0.05 \\
0.09 \\
0.08\end{array}$ & $\begin{array}{l}31077_{2}-42251_{3}^{\circ} \\
34465_{3}-45551_{3}^{\circ} \\
33201_{5}-43985^{\circ}{ }_{4} \\
32826_{3}-43514^{\circ} \\
37414_{3}-47483^{\circ} \\
37414_{3}-47442_{2}^{\circ}\end{array}$ \\
\hline
\end{tabular}

The new level at $33201.61(J=5)$ has no place in the $(5 d+6 s)^{6}$ theoretical scheme as given by Shadmi and Caspi. Since it makes 20 combinations (3 times above the noise level) it is probably real.

The lines classified by these new levels are listed in table 2. The line at $7098 \AA$ was inadvertently omitted from table 3 of Laun and Corliss [1968].

\section{References}

Laun, Donald D. and Corliss, C. H. (1968), J. Res. NBS 72 A (Phys. and Chem.) No. 6, 609 (1968).

Shadmi, Y. and Caspi, E. (1968), J. Res. NBS 72A(Phys. and Chem.) No. 6, 757 (1968).

(Paper 73A3-551) 